
\title{
BReserch Soute \\ Effect of Resilience on Stress, Anxiety and Depression in Civil Servants: The Mediating and Moderating Model
}

Huiling Song

Department of Children, Adolescents and Women Health, College of Public Health, Zhengzhou University Mengjie Zhang

Zhengzhou University

\section{Yanjie Wang}

Zhengzhou University

\section{Liying Yang}

Zhengzhou University

Yanyu Wang

Zhengzhou University

Yan Li ( $\nabla$ liyanzt@zzu.edu.cn )

Zhengzhou University https://orcid.org/0000-0002-5237-7569

\section{Research}

Keywords: Civil servants, Stress, Depression, Anxiety, Resilience

Posted Date: September 18th, 2020

DOI: https://doi.org/10.21203/rs.3.rs-76138/v1

License: () (1) This work is licensed under a Creative Commons Attribution 4.0 International License. Read Full License 


\section{Title page}

\section{Effect of resilience on stress, anxiety and depression in civil servants: the mediating and moderating model}

Huiling Song ${ }^{\text {a }}$, Mengjie Zhang ${ }^{a}$, Yanjie Wang ${ }^{a}$, Liying Yang ${ }^{\text {a }}$, Yanyu Wang ${ }^{\text {b }}$ Yan $\mathrm{Li}^{\mathrm{a}, \mathrm{c},{ }^{*}}$

a Department of Children, Adolescents and Women Health, College of Public Health, Zhengzhou University, Zhengzhou, Henan, China.

b The First Affiliated Hospital and College of Clinical Medicine of Henan University of Science and Technology, Luoyang, Henan, China. (24 Jinghua Road, Luoyang, 4571003, China )

c School of Physical Education (Main Campus), Zhengzhou University, Zhengzhou, Henan, China

${ }^{*}$ Corresponding author: Department of Children, Adolescents and Women Health, College of Public Health, Zhengzhou University, 100 Kexue Road, Zhengzhou, 450000, Henan, China

E-mail address: liyanzt@zzu.edu.cn (Yan Li) 


\section{Effect of resilience on stress, anxiety and depression in civil servants: the mediating and moderating model}

\section{Abstract}

Background: Civil servants are a special group in China, and there is limited research on how their resilience affects mental health, such as depression and anxiety. Therefore, the aim of the paper is to explore the role of resilience through the relationship between stress, depression and anxiety, and to further provide an explanation for how resilience interacts with stress, depression and anxiety in civil servants.

Method: In a cross-sectional survey, 302 civil servants completed a battery of questionnaires. The Civil Servants Stress Scale (CSSS) was used to assess the stress of civil servants. The depression and anxiety of participants were evaluated by the Self-rating Depression Scale (SDS) and the Self-rating Anxiety Scale (SAS), respectively. The Conner-Davidson Resilience Scale (CD-RISC) was used to assess the stress of civil servants as well. We conducted the moderating and mediating models to the resilience, depression and anxiety in civil servants.

Results: The results have shown that resilience was related to stress, depression, and anxiety in civil servants. Stress was positively correlated with resilience. On the contrary, stress was negatively associated with depression and anxiety. 
Conclusion: Resilience played the moderating and mediating roles for stress, depression and anxiety. Resilience can moderate the relationship between stress and depression, anxiety as a dynamic moderate mode. Improving resilience and reducing stress play a vital role in preventing depression and anxiety in civil servants.

Keywords: Civil servants; Stress; Depression; Anxiety; Resilience

\section{Background}

The goal of building a moderately prosperous society in all respects in China, with the implementation of the accountability system and the reform of rank promotion, led to more rigorous assessment standards and the rare chance of promotion in civil servants[1]. In this case, civil servants constantly faced the pressure of occupation, individual and society[2]. This reminded us that paying close attention to the mental health of civil servants is extremely essential. However, the mental health problems of civil servants have not fully been explored.

Past research had shown that long-term exposure to stress hormones throughout the lifespan can affect brain structures involved recognition and mental health[3]. Chronic stress may result in the increasing prevalence of mental disorders, chronic diseases $[4,5]$ and psychological problems are increased[6]. Although the pathogenesis of the depression and anxiety is not clear, some studies have found that there was a strong relationship between stress and depression[7], anxiety[8]. Furthermore, resilience has been found to 
mediate the associations between stress, depression, and anxiety among adolescents and the pregnant $[9,10]$. Also, resilience is an important protective factor that can moderate the development of psychiatric symptoms when an individual encounters some stressful events[11]. Civil servants face enormous pressure in work and life, which causes some psychological problems, such as depression, anxiety[12, 13]. Resilience can be regarded as a protective factor within individuals, which is of great significance to relieve pressure and promote mental health[14, 15]. Hence, we supposed that resilience can potentially improve coping strategies to avoid the negative effects of stress on mental health such as depression, anxiety among civil servants.

To data, the research on the impact of resilience on stress, depression and anxiety mainly focused on the adolescents and college students[16]. Stress and mental health in the civil servants are being increasingly recognized in China[17]. However, many studies provided results only from qualitative research studies or limited to stress with physical health[18, 19]. Little attention has been given to the interrelationships of mental health problems with stress in the civil servants[20].

The purpose of the study was to examine the moderating and mediating effects of resilience between stress and depression and anxiety in civil servants. The hypotheses were as follows: (1) Resilience will mediate the relationship between stress, depression and anxiety, respectively, and that (2) the relationship between stress, depression and anxiety will be 
modified by resilience. By exploring these hypotheses, we hope to understand the mechanisms of the influence of resilience on stress, depression and anxiety, and to further provide a scientific basis for the development and implementation of mental health improvement strategies for civil servants.

\section{Materials and methods}

\subsection{Participants}

The cross-sectional study enrolled 350 participants from a county in Henan. The study was conducted following the ethical principles of Zhengzhou University. Written informed consent was obtained from each participant before starting any investigation related study.

\subsection{Demographic data}

Demographic characteristics included gender, age, an only child, marital status, degree of education, seniority, position, relationship with colleagues, physical exercise, and monthly income.

\subsection{Civil Servants Stress Scale (CSSS)}

The CSSS is a 38-item self-report scale, which is divided into 6 dimensions as follows: which are management and development, life relationships, work relationships, health and responsibility, economic stress, and workload. The CSSS is scored on a 10-point with response categories ranging from 1 (no stress) to 10 (extremely stress). Total score ranges from 0 to 380 and the higher score indicates higher stress. The Cronbach'S a coefficient and Split-half reliability are 0.960 and 0.976 , respectively[21]. The Cronbach'S 
a coefficients of the 6 dimensions are $0.842 \sim 0.925$. The scale with good reliability and validity can be used to evaluate the overall stress situation of civil servants in China.

\subsection{Self-rating Depression Scale (SDS)}

The SDS[22] is a 20-item self-report scale, which is scored on a 4-point with ranging 1 (occasionally) from to 4 (constantly). Then add up the scores of 20 items to get the total rough score, and multiply the total rough score by 1.25 to get the standard score. The higher total standard scores indicate more severity of depression. The Cronbach'S a coefficient and Split-half reliability are 0.784 and 0.92 , respectively[23].

\subsection{Self-rating Anxiety Scale (SAS)}

The SAS[22] is a 20-item self-report scale, which is scored on a 4-point with ranging 1 (occasionally) from to 4 (constantly). Then add up the scores of 20 items to get the total rough score, and multiply the total rough score by 1.25 to get the standard score. The higher total standard score indicates more severity of anxiety. Cronbach'S a coefficient is 0.767 [24].

\subsection{Connor-Davidson Resilience Scale (CD-RISC)}

The CD-RISC is a 25 -item self-report scale, which is scored on a 5-point with ranging 1 (never) from to 5 (all the time). The higher score reflects greater resilience. The Cronbach'S a coefficient of the English version is 0.89 , and the coefficient of retest reliability is $0.87[25]$. The CD-RSCl scale with good reliability validity has been widely used in predicting mental resilience. 


\subsection{Statistical analyses}

All statistical analyses were performed using the SPSS software, and version 21.0. Descriptive statistics were the mean and standard deviation. T-test and Analysis of Variance (ANOVA) were used to compare the differences between depression and anxiety in the basic demographic data. Pearson correlations were used to explore associations among the main variables in the study. The moderating and mediating roles of resilience were analyzed in PROCESS using least squares regression[26].

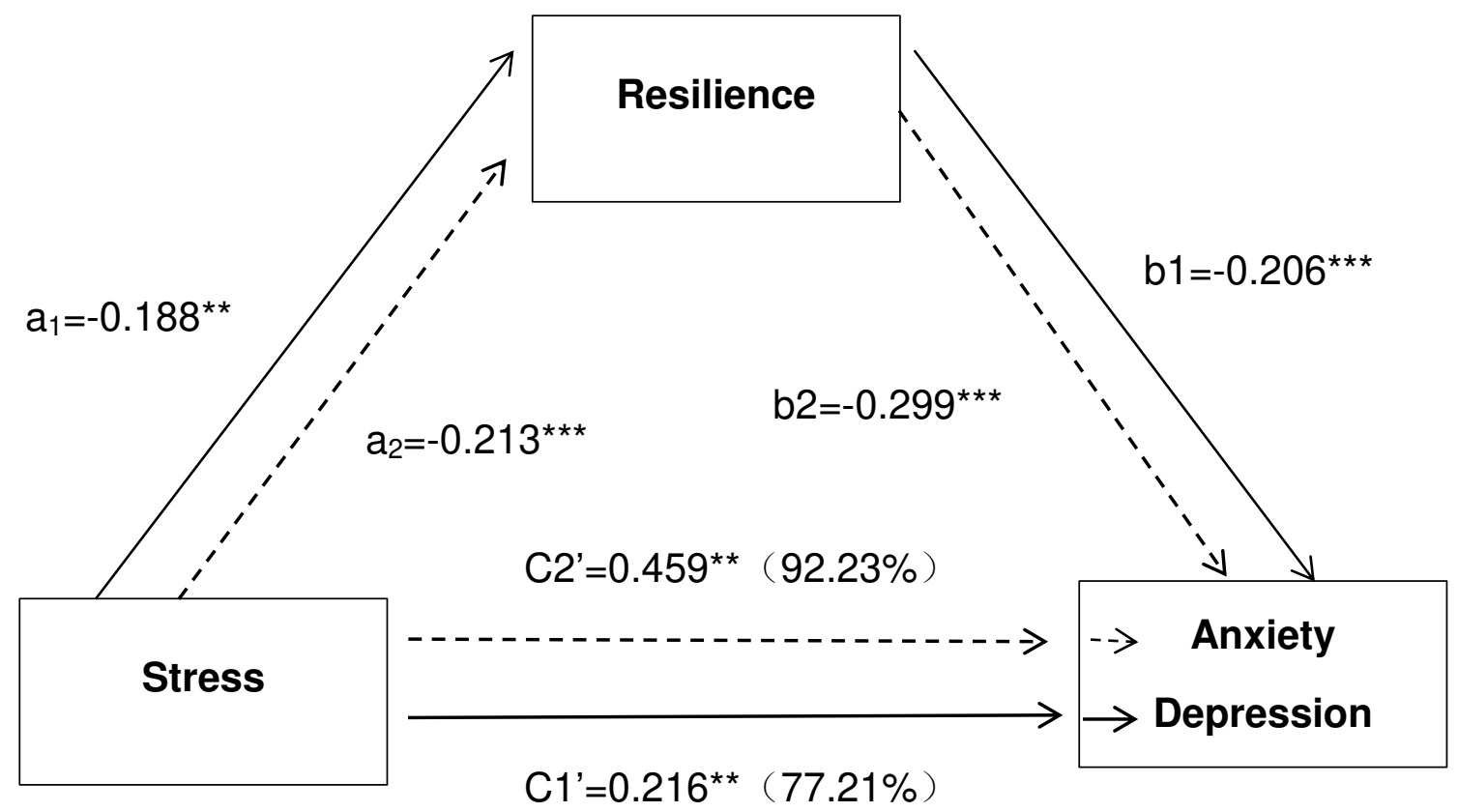

Figure1. Mediating effects of resilience in the relationship between stress(short for stress) and depression (solid line), anxiety (dash line) in civil servants ( $N=302)$.

Note: ${ }^{*} \mathrm{P}<05,{ }^{* *} \mathrm{P}<<01,{ }^{* * *} \mathrm{P}<001$.

\section{Results}

3.1 Demographic characteristics

The participants were aged between 17 and 56 years $(M=30.80, S D=7.85)$. 
There were 117 girls and 185 boys, and the sex ratio was close to 4:6. The differences between depressive, anxiety depression and anxiety in the demographic data were presented in table 1. There were significant differences in home address, degree of education, physical exercise, monthly income, and somatic diseases for depression $(P<0.05)$. There was a significant difference in the relationship with colleagues, physical exercise, monthly income for anxiety $(\mathrm{P}<0.05)$.

Table 1

The differences between depression and anxiety in the demographic data.

\begin{tabular}{|c|c|c|c|c|c|c|c|}
\hline \multirow{2}{*}{\multicolumn{2}{|c|}{ Variables }} & \multicolumn{3}{|c|}{ Depression } & \multicolumn{3}{|c|}{ Anxiety } \\
\hline & & Mean $\pm S D$ & $t / F$ & $P$ & Mean $\pm S D$ & $t / F$ & $P$ \\
\hline \multirow[t]{2}{*}{ Gender } & Man & $-0.08 \pm 0.96$ & -1.750 & 0.081 & $-0.06 \pm 0.93$ & -1.281 & 0.201 \\
\hline & Female & $0.13 \pm 1.05$ & & & $0.09 \pm 1.09$ & & \\
\hline \multirow[t]{3}{*}{ Age } & $\leq 30$ & $0.05 \pm 1.01$ & 1.545 & 0.215 & $0.06 \pm 1.07$ & 1.836 & 0.161 \\
\hline & $31 \sim 50$ & $-0.04 \pm 0.98$ & & & $-0.05 \pm 0.88$ & & \\
\hline & $\geq 51$ & $-0.60 \pm 0.80$ & & & $-0.62 \pm 0.72$ & & \\
\hline Home & City & $-0.13 \pm 0.97$ & 4.249 & 0.015 & $-0.08 \pm 1.02$ & 1.601 & 0.203 \\
\hline \multirow[t]{2}{*}{ address } & Town & $-0.03 \pm 0.91$ & & & $-0.02 \pm 0.87$ & & \\
\hline & Village & $0.26 \pm 1.07$ & & & $0.16 \pm 1.04$ & & \\
\hline \multirow[t]{2}{*}{ The one-child } & Yes & $0.91 \pm 0.10$ & 0.538 & 0.591 & $1.03 \pm 0.07$ & 0.672 & 0.502 \\
\hline & No & $0.96 \pm 0.11$ & & & $1.02 \pm 0.07$ & & \\
\hline \multirow[t]{3}{*}{ Marital status } & Unmarried & $0.01 \pm 1.01$ & 0.051 & 0.950 & $-0.04 \pm 0.95$ & 2.433 & 0.089 \\
\hline & Married & $-0.01 \pm 1.00$ & & & $0.04 \pm 1.02$ & & \\
\hline & Divorce & $-0.20 \pm 0.36$ & & & $-1.46 \pm 0.55$ & & \\
\hline \multirow[t]{3}{*}{ Education } & Junior college & $0.14 \pm 0.95$ & 3.685 & 0.026 & $-0.01 \pm 0.94$ & 1.799 & 0.167 \\
\hline & Undergraduate & $-0.10 \pm 1.03$ & & & $0.05 \pm 1.06$ & & \\
\hline & Postgraduate & $-0.54 \pm 0.97$ & & & $-0.57 \pm 0.80$ & & \\
\hline Seniority & $<1$ & $0.03 \pm 1.07$ & 0.779 & 0.506 & $0.01 \pm 1.04$ & 1.286 & 0.279 \\
\hline \multirow[t]{3}{*}{ (year) } & $1 \sim 5$ & $0.04 \pm 0.97$ & & & $-0.02 \pm 0.97$ & & \\
\hline & $6 \sim 10$ & $0.08 \pm 1.05$ & & & $0.20 \pm 1.16$ & & \\
\hline & $>10$ & $-0.15 \pm 0.98$ & & & $-0.14 \pm 0.89$ & & \\
\hline \multirow[t]{2}{*}{ Position } & Clerk & $0.03 \pm 0.98$ & 1.780 & 0.170 & $0.03 \pm 1.01$ & 1.832 & 0.162 \\
\hline & Section-level & $-0.35 \pm 1.13$ & & & $-0.35 \pm 0.80$ & & \\
\hline
\end{tabular}




\begin{tabular}{|c|c|c|c|c|c|c|c|}
\hline \multirow{3}{*}{$\begin{array}{l}\text { Relationship } \\
\text { with }\end{array}$} & $\begin{array}{l}\text { cadres } \\
\text { Office-level } \\
\text { cadres }\end{array}$ & \multicolumn{3}{|l|}{$-0.20 \pm 1.26$} & $-0.18 \pm 1.10$ & & \\
\hline & Good & $-0.01 \pm 1.01$ & 1.047 & 0.352 & $-0.02 \pm 0.99$ & 3.098 & 0.047 \\
\hline & Average & $0.02 \pm 0.97$ & & & $0.02 \pm 1.01$ & & \\
\hline coworkers & Poor & $1.00 \pm 0.45$ & & & $1.72 \pm 0.32$ & & \\
\hline Physical & Often & $0.09 \pm 1.02$ & 3.586 & 0.029 & $-0.14 \pm 0.77$ & 4.599 & 0.011 \\
\hline \multirow[t]{2}{*}{ exercise } & By chance & $-0.13 \pm 0.92$ & & & $-0.08 \pm 0.90$ & & \\
\hline & Never & $0.23 \pm 1.12$ & & & $0.31 \pm 1.30$ & & \\
\hline Monthly & $\leq 1500$ & $0.39 \pm 0.87$ & 7.013 & $<0.001$ & $0.47 \pm 1.23$ & 4.942 & 0.002 \\
\hline \multirow[t]{3}{*}{ income(CNY) } & $1501 \sim 2499$ & $0.22 \pm 1.01$ & & & $0.16 \pm 0.98$ & & \\
\hline & $2500 \sim 3499$ & $-0.13 \pm 0.92$ & & & $-0.18 \pm 0.96$ & & \\
\hline & $\geq 3500$ & $-0.35 \pm 0.98$ & & & $-0.22 \pm 0.93$ & & \\
\hline Somatic & Yes & $0.51 \pm 1.17$ & 2.225 & 0.027 & $-0.03 \pm 0.98$ & 0.993 & 0.322 \\
\hline diseases & No & $0.23 \pm 1.07$ & & & $1.00 \pm 0.06$ & & \\
\hline
\end{tabular}

SD: standard deviation.

In table 2, stress was positively correlated with depression and anxiety $(P<0.001)$. Conversely, stress was negatively correlated with resilience $(P<0.001)$. Resilience was negatively correlated with depression and anxiety $(P<0.001)$.

Table 2

Pearson correlations among the target and the control variables $(r)$

\begin{tabular}{lllll}
\hline Variables & Mean \pm SD & 1 & 2 & 3 \\
\hline 1.stress & $149.52 \pm 65.89$ & & & \\
2.depression & $0.54 \pm 0.10$ & $0.212^{\star * *}$ & & \\
3.anxiety & $48.23 \pm 11.17$ & $0.444^{\star \star *}$ & $0.699^{\star * *}$ & \\
4.resilience & $48.06 \pm 9.32$ & $-0.212^{\star \star *}$ & $-0.382^{\star \star *}$ & $-0.343^{\star * *}$ \\
\hline
\end{tabular}

SD: standard deviation.

Note: $\mathrm{P}<0.05,{ }^{* *} \mathrm{P}<0.01,{ }^{* *} \mathrm{P}<0.001$

As shown in Table 3, two mediation analyses were performed. The depression (as dependent variable), home address, degree of education, physical exercise, monthly income, oneself has an illness (as covariates), 
stress (as independent variables), and resilience (as mediation) entered into model 59. The results indicated that resilience partially mediated the relationship between stress and depression. The direct effect is $77.21 \%$ and the indirect effect is $22.79 \%$. Secondly, the anxiety (dependent variable), relationship with colleagues, physical exercise, monthly income (covariates), stress (independent variables) and resilience (mediation) were entered. The resilience had made mediation effect between stress and anxiety. The direct effect is $92.23 \%$ and the indirect effect is $7.77 \%$.

Table3

Mediating effects of resilience in the relationship between stress and depression, anxiety

\begin{tabular}{|c|c|c|c|c|c|c|}
\hline \multirow{2}{*}{ Effect } & \multicolumn{3}{|c|}{ Depression } & \multicolumn{3}{|c|}{ Anxiety } \\
\hline & $\beta(\mathrm{SE})$ & $P$ & $95 \% \mathrm{Cl}$ & $\beta(\mathrm{SE})$ & $P$ & $95 \% \mathrm{Cl}$ \\
\hline$a$ & $-0.22(0.57)$ & $<0.001$ & & $-0.19(0.06)$ & 0.001 & \\
\hline b & $-0.30(0.05)$ & $<0.001$ & & $-0.21(0.05)$ & $<0.001$ & \\
\hline$C^{\prime}$ & $0.22(0.05)$ & $<0.001$ & & $0.46(0.05)$ & $<0.001$ & \\
\hline$a * b$ & $0.06(0.02)$ & & $(0.020,0.115)$ & $0.04(0.02)$ & & $(0.009,0.078)$ \\
\hline
\end{tabular}

As presented in table 4, two moderation analyses were performed. The depression (dependent variable), home address, degree of education, physical exercise, monthly income, oneself has an illness (covariates), stress (independent variables) and resilience (moderator) entered into model 59. The interaction effects of stress and resilience on depression were significant $(P<0.001)$. Secondly, the anxiety (dependent variable), relationship with colleagues, physical exercise, monthly income (covariates), stress (independent variables) and resilience (moderation) were entered. The 
interaction effects of stress and resilience on anxiety were significant $(P<0.001)$. Thus, the hypotheses that resilience moderate between stress, depression and anxiety is valid.

Table 4

Moderating effects of resilience in the relationship between stress and depression, anxiety

\begin{tabular}{|c|c|c|c|c|c|c|c|c|}
\hline \multirow[b]{2}{*}{ Predictors } & \multicolumn{4}{|c|}{ Depression } & \multicolumn{4}{|c|}{ Anxiety } \\
\hline & $\beta(\mathrm{SE})$ & $P$ & $R^{2}$ & $F$ & $\beta(\mathrm{SE})$ & $P$ & $R^{2}$ & $F$ \\
\hline Stress & $0.23(0.05)$ & $<0.001$ & 0.280 & 14.271 & $0.47(0.05)$ & $<0.001$ & 0.358 & 23.396 \\
\hline Resilience & $-0.34(0.05)$ & $<0.001$ & & & $-0.25(0.05)$ & $<0.001$ & & \\
\hline Stress $\times$ Resilience & $-0.13(0.04)$ & 0.001 & & & $-0.13(0.04)$ & $<0.001$ & & \\
\hline
\end{tabular}

To further analyze the moderating effect of resilience on the relationship between depression and anxiety, two simple slope analyses were performed. Stress and resilience were classified as high $(M+S D)$ and low (M-SD). Firstly, the dependent variable was depression as showing in Fig 2. The estimates of 95\% bias-corrected bootstrap Cl of M-SD and M+SD, were $(0.2231,0.4936)$ and $(-0.0337,0.2219)$, respectively. The difference of $95 \%$ bias-corrected bootstrap $\mathrm{Cl}$ of $\mathrm{M}-\mathrm{SD}$ and $\mathrm{M}+\mathrm{SD}$ is $(0.2568,0.2712)$. There was no zero in the difference of $95 \%$ bias-corrected bootstrap $\mathrm{Cl}$ indicated that resilience has made a moderation effect between stress and depression. Secondly, the dependent variable was anxiety as shown in Fig 3. The estimates of $95 \%$ bias-corrected bootstrap $\mathrm{Cl}$ of $\mathrm{M}-\mathrm{SD}$ and $\mathrm{M}+\mathrm{SD}$, were $(0.4754,0.7337)$ and $(0.2219,0.4613)$, respectively. The difference of $95 \%$ bias-corrected bootstrap $\mathrm{Cl}$ of M-SD and M+SD is $(0.2535,0.2724)$. There is no zero in the difference of 95\% bias-corrected bootstrap $\mathrm{Cl}$ indicated that resilience has made a 
moderation effect between stress and anxiety.

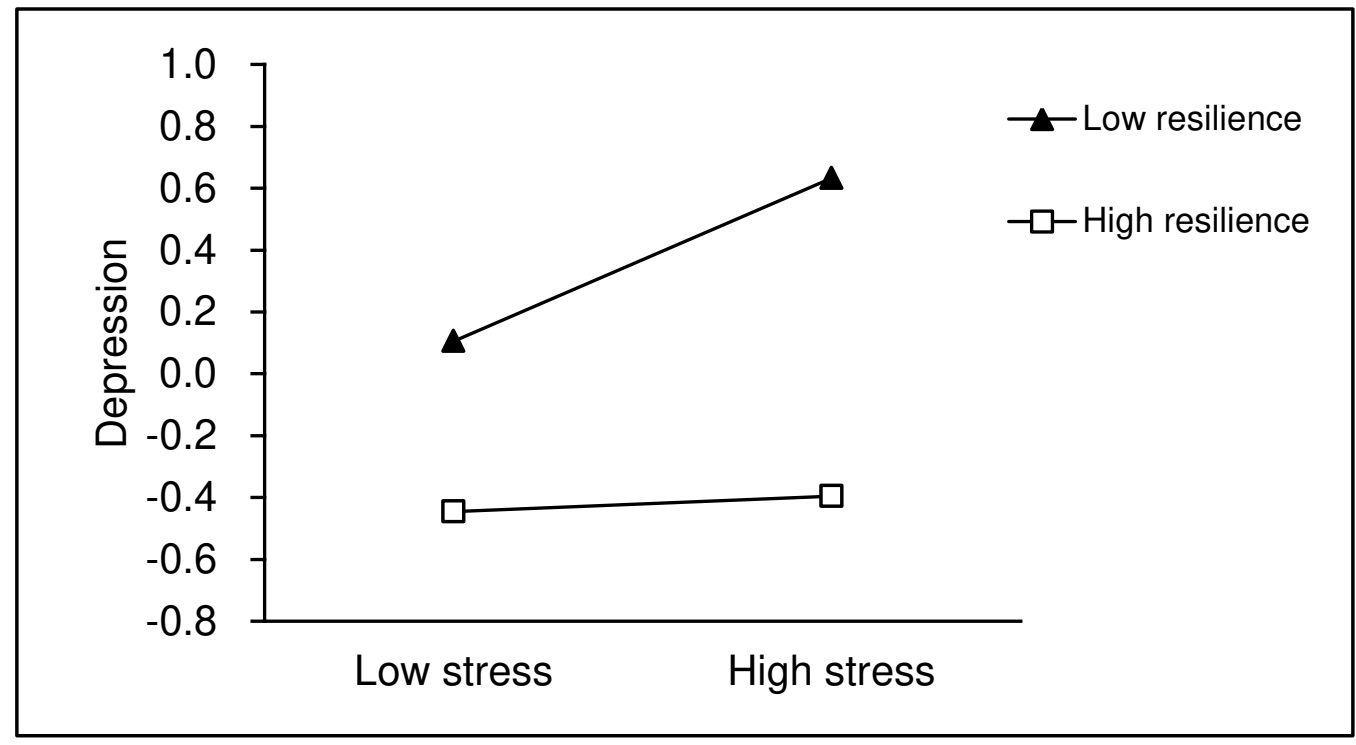

Figure 2. Standardized simple regression lines for the association between stress and depression at low (one SD below the mean) and high resilience (one SD high the mean).

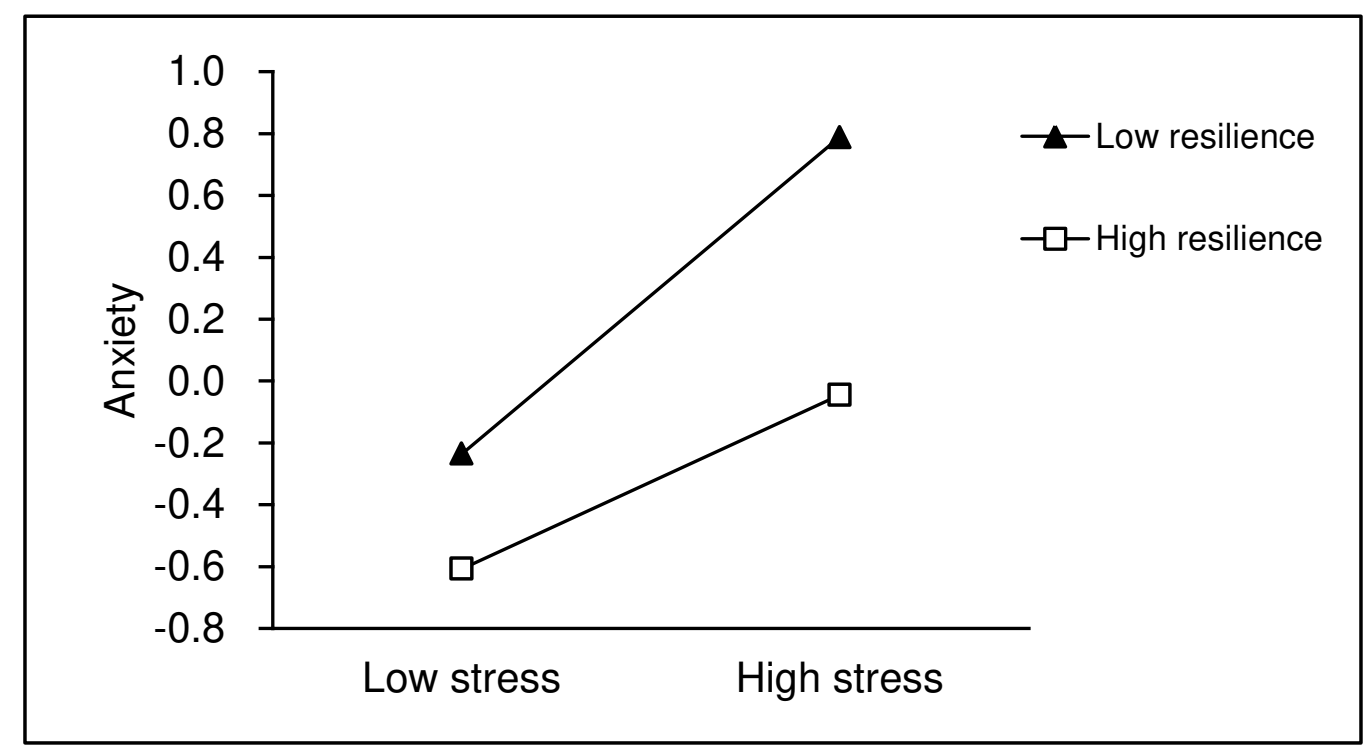

Figure 3. Standardized simple regression lines for the association between stress and anxiety at low (one SD below the mean) and high resilience (one SD high the mean).

\section{Discussion and Limitations}

In this study, we reported that depression was related to the home address, degree of education, physical exercise, monthly income and 
somatic diseases among civil servants. This study also showed that there was a significant difference in the relationship with colleagues, physical exercise, monthly income for anxiety in civil servants. Previous studies have suggested that home address, degree of education, physical exercise, monthly income, relationship with colleagues and somatic diseases have an effect on depression and anxiety in different groups of people from students to commuters[27-29]. In this study, we focused on this special group of civil servants. In China, civil servants have a strict promotion system, and degree of education plays an important role in promotion. The high education level of civil servants has certain advantages in the promotion and social welfare, while those with lower education level has relatively high professional competitive pressure, which leads to higher depression level[4]. In addition, there were significant differences in depression and anxiety among civil servants who exercised at different frequencies, which is related to the positive impact of exercise on mood states such as depression and anxiety, through physiological and biochemical mechanisms $[4,13]$.

As expected, stress of civil servants is positively correlated with depression and anxiety, while resilience is negatively corrected with stress, depression and anxiety, which indicated that stress in civil servants is a risk factor for depression and anxiety, while resilience is a protective factor for depression and anxiety. This is consistent with previous studies of 
depression and anxiety[9, 12, 13, 15]. Stress is a risk factor for depression and anxiety. To some extent, stress can cause and aggravate depression and anxiety. It can also be said that civil servants with depression and anxiety, to a certain extent, have relatively low ability to face and negotiate pressure to overcome adverse consequences. Furthermore, resilience is a protective factor for depression and anxiety. The hypothesis that resilience modified the relationship between stress, depression and anxiety has been confirmed. To some extent, resilience can relieve depression and anxiety caused or aggravated by stress. Interestingly, civil servants with high resilience may have more ability to deal with the stress in life and work than those with low resilience, thereby reducing the risk of depression and anxiety. Previous studies have also found that resilience can partially moderate stress and other psychological and emotional problems[14, 17].

Through moderating and mediating analysis, it is found that stress can not only directly affect mental health, causing depression and anxiety, but also indirectly affect mental health level of civil servants through resilience. Resilience can directly block the negative impact of stress on mental health and thus become a protective factor of mental health under stress. The reason why resilience can be an intrinsic protective factor may be that individuals with higher resilience are better at assessing stressful events in a positive cognitive way in the face of the same stress. They tend to adjust the relationship between the environment and individuals more actively, 
stimulate and promote their own potential, make full use of various resources, face pressure, and achieve a good state of adaptation and development. This is also consistent with the results of previous resilience model studies[14, 17].

The mediation analysis found that resilience had more than three times of the effect on depression than anxiety, suggesting that resilience was more important than anxiety in explaining depression. The simple slope analysis showed that the effect of stress on depression of civil servants with higher resilience scores was lower, while those with lower resilience scores were significantly higher in the relationship between stress and depressive. Civil servants in the high resilience group scores were lower on the relationship between stress and anxiety than those in the low resilience group. In the present study, resilience accounting for an important part of the variation in anxiety but even larger variations in depression may suggest interventions should target ways to enhance resilience and at the same time minimize the effects of stress. When considering interventions that promote resilience, it may be more relevant to civil servants who show anxiety and depression as a result of the direct effects of stress.

There are some limitations to this study. First, it is a cross-sectional study, which means that causality cannot be determined. Second, because of the self-reporting questionnaire used in the current study, the results may 
be constrained by memory bias and individual subjective consciousness. Conclusion

\section{Conclusion}

Resilience can moderate the relationship between stress and depression, anxiety as a dynamic moderate mode. Improving resilience and reducing stress play a vital role in preventing depression and anxiety in civil servants. The future study can explore how to enhance the resilience of civil servants and the mechanism and process of resilience.

\section{List of abbreviations}

\begin{tabular}{|c|c|}
\hline Abbreviation & Full name \\
\hline CSSS & Civil Servants Stress Scale \\
\hline SDS & Self-rating Depression Scale \\
\hline SAS & Self-rating Anxiety Scale \\
\hline CD-RISC & Conner-Davidson Resilience Scale \\
\hline
\end{tabular}

\section{Declarations}

This study was supported by the Department of Children, Adolescents and Women Health, College of Public Health of Zhengzhou University in China. The author appreciates the help of editing and revisions from Mengjie Zhang and Yanjie Wang in the paper. The author also appreciates all the participants and Liying Yang and Yanyu Wang of the study for their help in recruiting participants and collecting questionnaires. All acknowledge individuals have no conflicts of interest to declare. 


\section{References}

1. Liu, J., Rank promotion in civil servants promotion. Forum for party and government officials in China, 2019. 05: p. 26-29.

2. L, Z., et al., Correlations among Work Stressors, Work Stress Responses, and Subjective Well-Being of Civil Servants: Empirical Evidence from China. Iranian journal of public health, 2019. 48(6): p. 1059-1067

3. SJ, L., et al., Effects of stress throughout the lifespan on the brain, behaviour and cognition. Nature reviews. Neuroscience, 2009. 10(6): p. 434-45.

4. K, K., Measuring the effects of stress on the cardiovascular system during a disaster: the effective use of self-measured blood pressure monitoring. Journal of hypertension, 2010. 28(4): p. 657-9.

5. T, T. and S. M, Job dissatisfaction as a contributor to stress-related mental health problems among Japanese civil servants. Industrial health, 2013. 51(3): p. 307-18.

6. $\mathrm{H}, \mathrm{Y}$., et al., The impact of stress on body function: A review. EXCLI journal, 2017. 16: p. 1057-1072.

7. R, W., Can Hostile Attitudes and Hostile Affections Lead to Depressive Symptoms Under Stress? A Study Based on Racial/Ethnic and Gender Differences. Journal of racial and ethnic health disparities, 2020.

8. $\mathrm{CE}, \mathrm{H}$. , et al., The impact of life stress on adult depression and anxiety is dependent on gender and timing of exposure. Development and psychopathology, 2017. 29(4): p. 1443-1454.

9. F, A. and H. O, Adolescent stress and symptoms of anxiety and depression: Resilience explains and differentiates the relationships. Journal of affective disorders, 2016. 203: p. 213-220. 
10. $\mathrm{X}, \mathrm{M}$., et al., The impact of resilience on prenatal anxiety and depression among pregnant women in Shanghai. Journal of affective disorders, 2019. 250: p. 57-64.

11. Hjemdal, O., et al., Resilience predicting psychiatric symptoms: A prospective study of protective factors and their role in adjustment to stressful life events. Clinical Psychology \& Psychotherapy, 2006. 13(3): p. 194-201.

12. S, G., et al., Effect of Job Strain on Job Burnout, Mental Fatigue and Chronic Diseases among Civil Servants in the Xinjiang Uygur Autonomous Region of China. International journal of environmental research and public health, 2017. 14(8).

13. D, N., et al., Consequences of Job Insecurity on the Psychological and Physical Health of Greek Civil Servants. BioMed research international, 2015. 2015: p. 673623.

14. R, M., et al., Resilience or hope? Incremental and convergent validity of the resilience scale for adults (RSA) and the Herth hope scale (HHS) in the prediction of anxiety and depression. BMC psychology, 2017. 5(1): p. 36.

15. N, O.-B. and M. P, Psychological resilience and secondary traumatic stress in nurses working with terminally ill patients-The mediating role of job burnout. Psychological services, 2020.

16. Y, G., et al., Personality traits and depressive symptoms: The moderating and mediating effects of resilience in Chinese adolescents. Journal of affective disorders, 2020. 265: p. 611-617.

17. C, Z., et al., Relationships of mental health problems with stress among civil servants in Guangzhou, China. Community mental health journal, 2014. 50(8): p. 991-6.

18. L, W., [An investigation of occupational stress, social support, and happiness of junior civil servants]. Zhonghua lao dong wei sheng zhi ye bing za zhi = Zhonghua laodong weisheng 
zhiyebing zazhi $=$ Chinese journal of industrial hygiene and occupational diseases, 2015. 33(10): p. 753-6.

19. PY, L., et al., Environmental tobacco smoke (ETS) and hyperlipidemia modified by perceived work stress. PloS one, 2020. 15(1): p. e0227348.

20. W, K., et al., Mediating role of occupational stress and job satisfaction on the relationship between neuroticism and quality of life among Chinese civil servants: a structural equation model. Health and quality of life outcomes, 2020. 18(1): p. 34.

21. Development and evaluation of the Civil Servants Stress Scale.

22. ZUNG, W.W.K., A Self-Rating Depression Scale. Archives of General Psychiatry, 1965. 12(1): p. 63-70.

23. Zhang, M., Handbook of rating scales in psychiatry. Hunan science and technology press, 1993: p. 34-41.

24. Zhang, M., Handbook of rating scales in psychiatry. Hunan science and technology press, 1998: p. 35-42.

25. Connor, K.M. and J.R. Davidson, Development of a new resilience scale: the Connor-Davidson Resilience Scale (CD-RISC). Depress Anxiety, 2003. 18(2): p. 76-82.

26. Hayes , A.F., Introduction to Mediation, Moderation, and Conditional Process Analysis: A Regression - Based Approach. Journal of Educational Measurement, 2013. 3(51): p. 335-337.

27. C, L., et al., Prevalence and associated factors of depression and anxiety among doctoral students: the mediating effect of mentoring relationships on the association between research self-efficacy and depression/anxiety. Psychology research and behavior management, 2019. 12: p. 195-208. 
28. LB, F., et al., Work and home stress: associations with anxiety and depression symptoms. Occupational medicine (Oxford, England), 2015. 65(2): p. 110-6.

29. R, S., et al., Prevalence of depression and anxiety and correlations between depression, anxiety, family functioning, social support and coping styles among Chinese medical students. BMC psychology, 2020. 8(1): p. 38. 


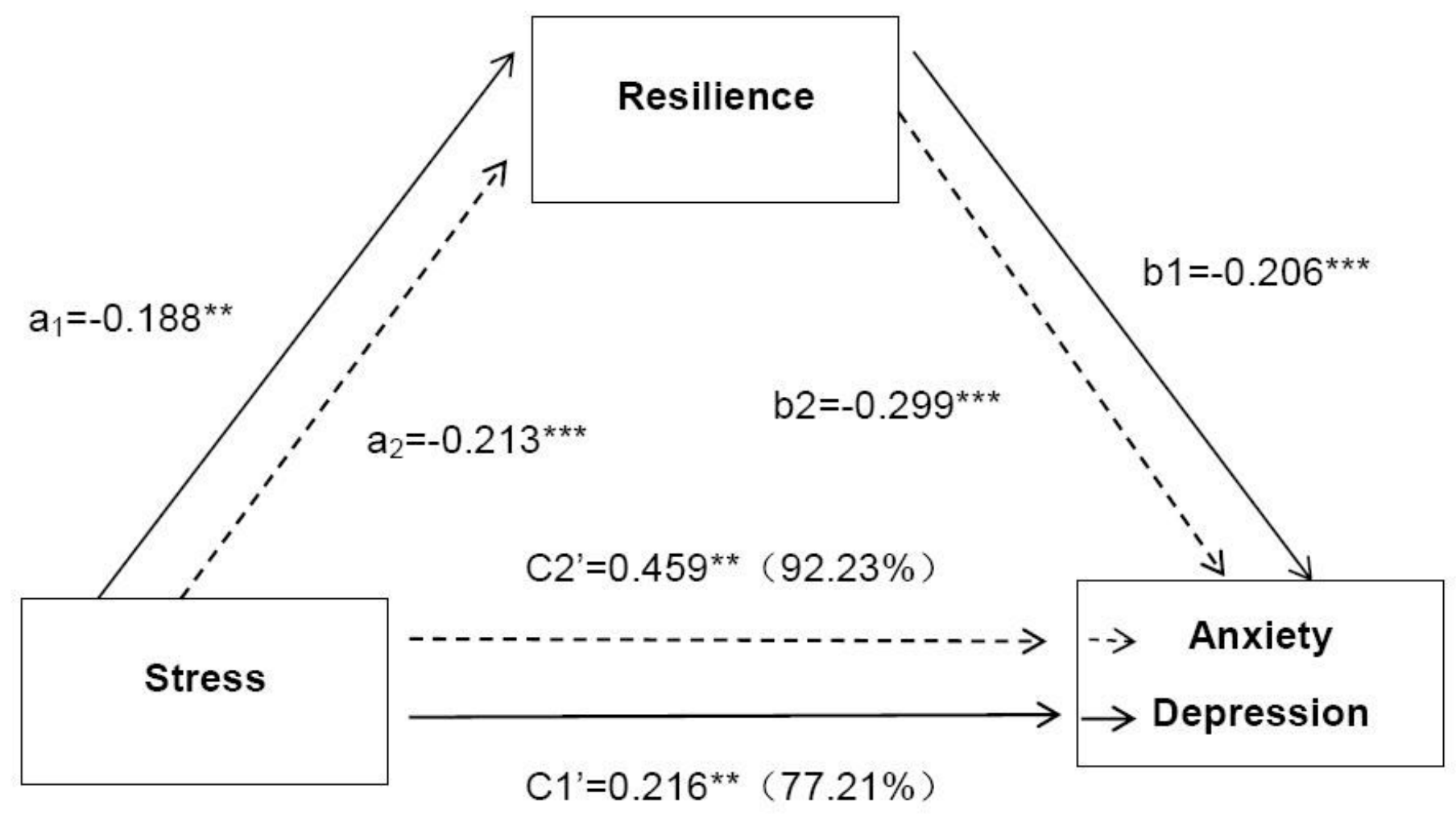

Figure 1

Mediating effects of resilience in the relationship between stress (short for stress) and depression (solid line), anxiety (dash line) in civil servants $(N=302)$. Note: ${ }^{*} P<05,{ }^{\star} * P<<01, * \star * P<001$. 


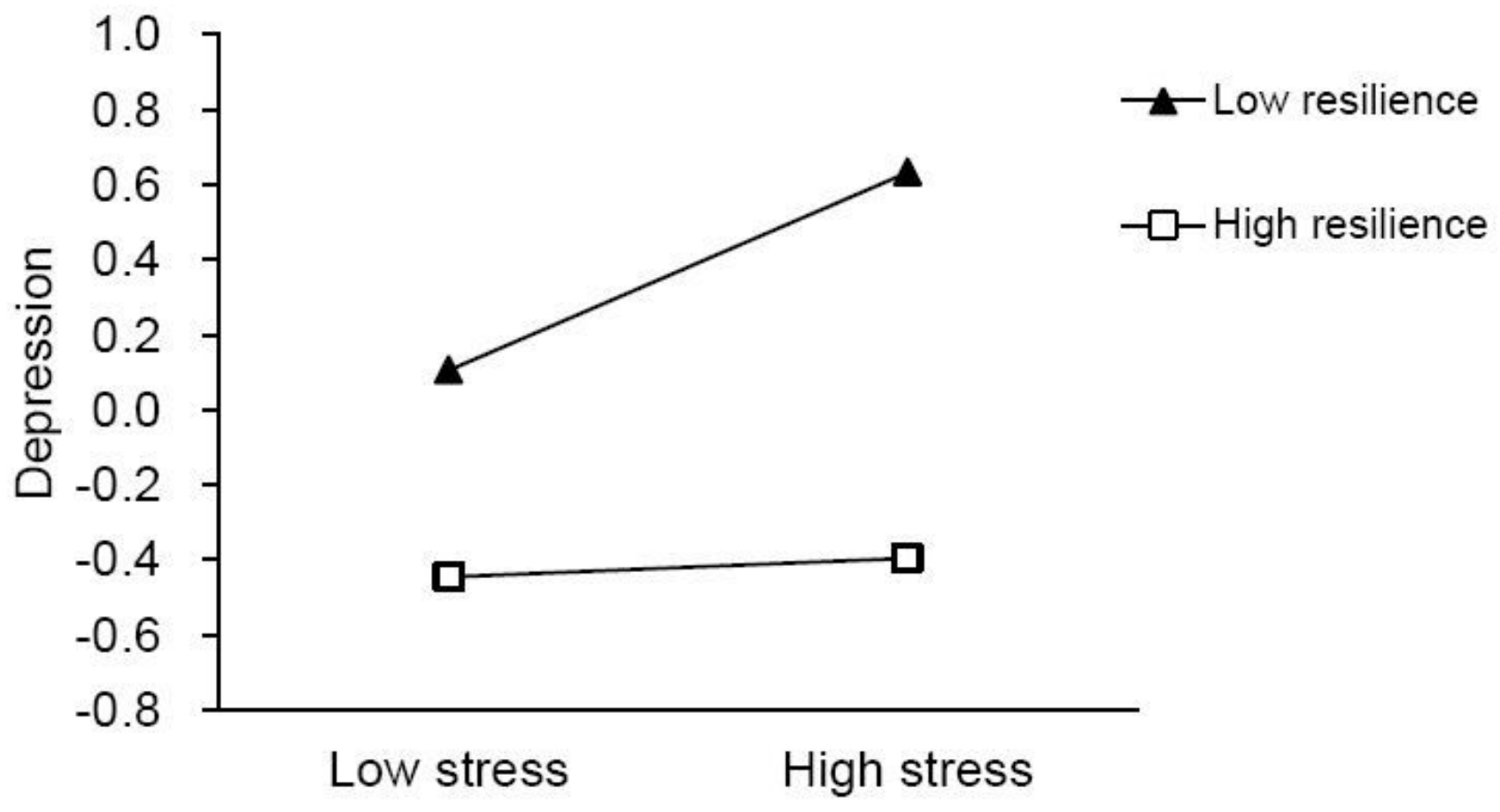

Figure 2

Standardized simple regression lines for the association between stress depression at low (one SD below the mean) and high resilience (one SD high the mean).

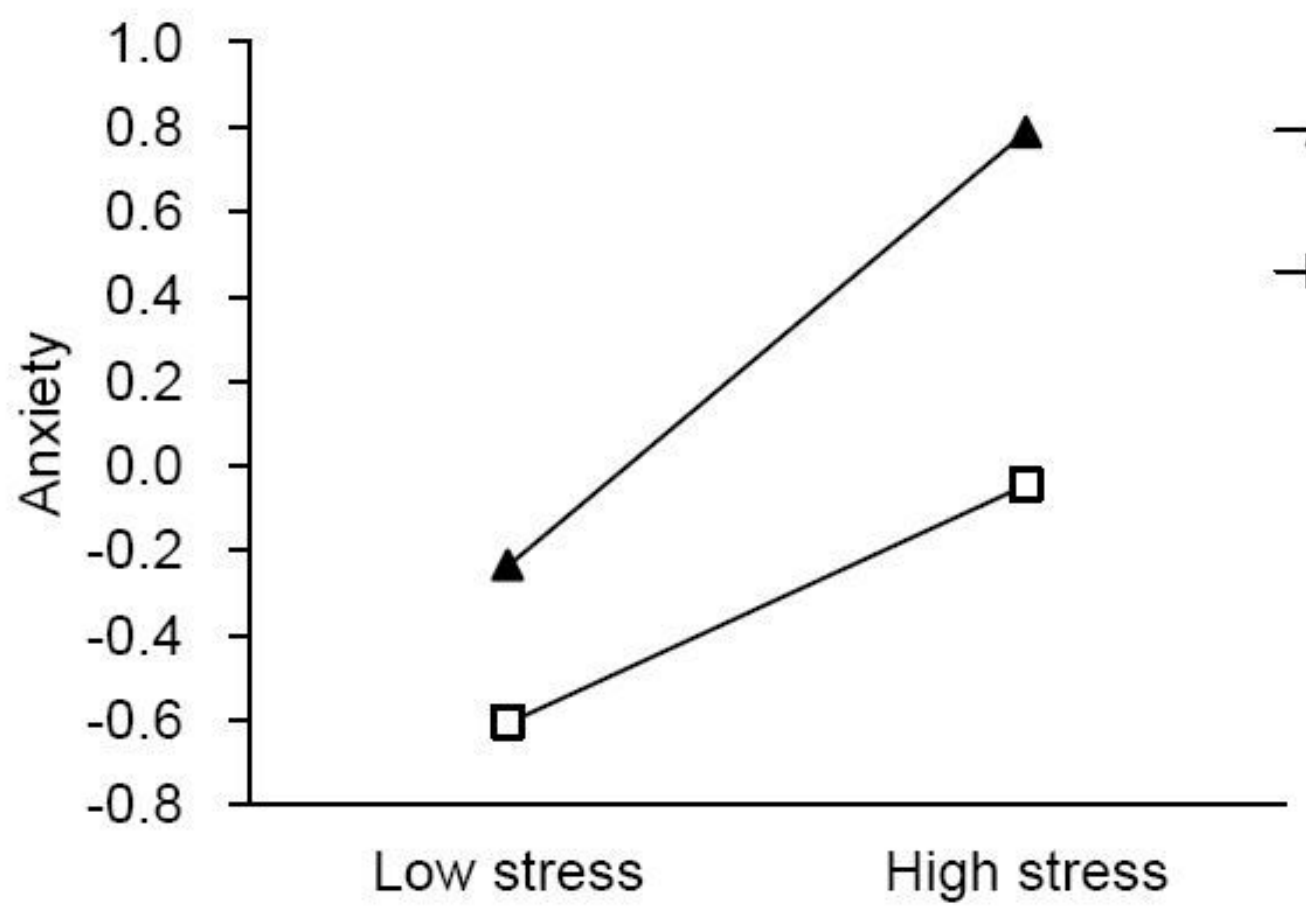

$\neg$ Low resilience

$-\square-$ High resilience 


\section{Figure 3}

Standardized simple regression lines for the association between stress anxiety at low (one SD below the mean) and high resilience (one SD high the mean). 